\title{
Single Center Trends in Acute Coronary Syndrome Volume and Outcomes During the COVID-19 Pandemic
}

\author{
Weiyi Tan ${ }^{\mathrm{a}}$, Rushi V. Parikh ${ }^{\mathrm{a}}$, Rebecca Chester ${ }^{\mathrm{a}}$, Jeffrey Harrell ${ }^{\mathrm{a}}$, Vanessa Franco ${ }^{\mathrm{b}}$, \\ Olcay Aksoy ${ }^{a}$, Ravi Dave ${ }^{a}$, Asim Rafique ${ }^{a}$, Marcella Press ${ }^{a, c}$
}

\begin{abstract}
Background: The coronavirus disease 2019 (COVID-19) pandemic has greatly affected healthcare delivery across the world. In this report, we aim to further characterize the changes in cardiac catheterization at our institution, specifically in the setting of acute coronary syndrome (ACS).

Methods: We performed a retrospective analysis of patients undergoing cardiac catheterization between December 23, 2019 and April 12,2020 at our institution. All patients with cardiac catheterizations for ACS, ST-elevation myocardial infarction (STEMI) activation, and out-of-hospital cardiac arrest (OHCA) were analyzed. Cardiac catheterization volume, as well as clinical and procedural characteristics of patients undergoing cardiac catheterization, was compared before and during the COVID-19 pandemic.
\end{abstract}

Results: Patients presenting with ACS and OHCA were similar in terms of demographics and comorbidities during both time periods. The mean monthly volume for ACS cases dropped by $26 \%$ during the pandemic, which was consistent among both unstable angina/nonST-elevation myocardial infarction (UA/NSTEMI) and STEMI cases. OHCA volume decreased significantly as well (five cases per month before to zero cases during the pandemic, $\mathrm{P}=0.01$ ). Among patients with STEMI, initial markers of cardiac injury, door-to-balloon time, and all-cause mortality were similar in both time periods.

Conclusions: With the start of the COVID-19 pandemic, there was a reduction in cardiac catheterization volume across the spectrum of ACS at our institution, which was consistent with reports from other centers across the globe. Patients with STEMI during the initial phase of the COVID-19 pandemic did not seem to have delays in presentation or significant differences in all-cause mortality at our institution.

Manuscript submitted May 9, 2020, accepted May 14, 2020

Published online June 3, 2020

aDivision of Cardiology, University of California, Los Angeles, CA, USA bepartment of Emergency Medicine, University of California, Los Angeles, CA, USA

${ }^{\mathrm{c}}$ Corresponding Author: Marcella Press, Division of Cardiology, University of California, Suite 630 West, 100 Medical Plaza, Los Angeles, CA 90095, USA. Email: MCalfonPress@mednet.ucla.edu

doi: https://doi.org/10.14740/cr1096
Keywords: COVID-19; STEMI; Acute coronary syndrome; Cardiac catheterization

\section{Introduction}

The coronavirus disease 2019 (COVID-19) pandemic has greatly affected healthcare delivery across the world, and many cardiac catheterization laboratories in the USA have scaled down the number of cases in accordance with regulatory and societal guidelines [1]. Recently published data reflect this reduction in catheterization volume and suggest a marked drop in ST-segment elevation myocardial infarction (STEMI) activations $[2,3]$. In the present report, we aim to further characterize these changes at our institution, specifically in the setting of acute coronary syndrome (ACS).

\section{Materials and Methods}

We performed a retrospective analysis of a cohort of patients undergoing cardiac catheterization between December 23, 2019 and April 12, 2020 at the University of California, Los Angeles (UCLA). We designated March 16, 2020 as the start of the COVID-19 era since UCLA stopped elective procedures on that date, and the "Safer at Home" order in Los Angeles was instituted soon thereafter on March 19, 2020. We included all patients with cardiac catheterizations for STEMI activations, ACS (i.e., unstable angina (UA), non-ST-segment elevation myocardial infarction (NSTEMI), true STEMI), and out-ofhospital cardiac arrest (OHCA). A STEMI activation was defined as a catheterization for a presumed STEMI or OHCA. A true STEMI was defined by the presence of a culprit lesion on angiogram. Characteristics of the true STEMI cases, including death, door-to-balloon (DTB) time, thrombolysis in myocardial infarction (TIMI) grade flow before and after the coronary intervention, TIMI thrombus grade, evidence of no reflow phenomenon, initial laboratory values for troponin-I and brain natriuretic peptide (BNP), and Q-waves on initial presenting electrocardiogram were recorded.

We compared cardiac catheterization volume for each clinical scenario (ACS, UA/NSTEMI, STEMI activations, STEMI, and OHCA) for the time period before and the month 
Table 1. Clinical Characteristics of Patients Undergoing Urgent or Emergent Cardiac Catheterization for Acute Coronary Syndrome or Out-of-Hospital Cardiac Arrest in the Time Period Before the COVID-19 Pandemic (December 23, 2019 to March 15, 2020), and During the COVID-19 Pandemic (March 16, 2020 to April 12, 2020)

\begin{tabular}{|c|c|c|}
\hline & Pre-COVID-19a $(\mathrm{N}=167)$ & COVID-19b $(N=37)$ \\
\hline \multicolumn{3}{|l|}{ Baseline clinical characteristics } \\
\hline Age (years, SD) & $63.6 \pm 13$ & $65.2 \pm 12.6$ \\
\hline History of hypertension & $66 \%$ & $59 \%$ \\
\hline History of coronary artery disease & $40 \%$ & $35 \%$ \\
\hline History of hyperlipidemia & $46 \%$ & $49 \%$ \\
\hline History of chronic kidney disease & $21 \%$ & $16 \%$ \\
\hline \multicolumn{3}{|l|}{ Presentation } \\
\hline UA/NSTEMI & $63 \%$ & $70 \%$ \\
\hline STEMI & $20 \%$ & $22 \%$ \\
\hline
\end{tabular}

aFrom December 23, 2019 to March 15, 2020. ' $F$ From March 16, 2020 to April 12, 2020. COVID-19: coronavirus disease 2019; SD: standard deviation; UA: unstable angina; NSTEMI: non-ST-elevation myocardial infarction; STEMI: ST-elevation myocardial infarction; OHCA: out-of-hospital cardiac arrest.

during the COVID-19 pandemic, and also compared the presentation of the true STEMI cases during these two periods. We performed independent $t$-tests for continuous variables and Pearson's Chi-squared tests for categorical variables. Stata 15.1 (College Station, Texas) was used for all statistical analyses and a $\mathrm{P}$ value $<0.05$ was considered statistically significant. The UCLA Institutional Review Board approved the study and waived patient consent due to the retrospective nature of the study.

\section{Results}

A total of 204 patients were included in this study (167 preCOVID-19 and 37 during the COVID-19 era). The clinical characteristics of both groups of patients were fairly similar; both groups of patients undergoing cardiac catheterization for urgent/emergent indications were mostly older males, many of whom had medical comorbidities (Table 1). The mean monthly ACS cases dropped by $26 \%(\mathrm{P}=0.09)$, which was consistent among both UA/NSTEMI $(26 \%, \mathrm{P}=0.12)$ and STEMI $(27 \%$, $\mathrm{P}=0.20$ ) (Fig. 1). The number of STEMI activations significantly dropped by $45 \%(\mathrm{P}=0.04)$. OHCA cases decreased from five cases per month pre-COVID-19 to no case thus far in the COVID-19 era $(\mathrm{P}=0.01)$.

For true STEMI cases, the DTB times remained similar (80.6 min pre-COVID-19 vs. 79.3 min during the COVID-19 era, $\mathrm{P}=0.47$ ). Initial markers of cardiac injury were not significantly higher in the COVID-19 era than the pre-COVID-19 era (troponin-I: $13.9 \mathrm{ng} / \mathrm{mL}$ vs. $9.9 \mathrm{ng} / \mathrm{mL}, \mathrm{P}=0.685$, and BNP 1,040 pg/mL vs. 348 pg/mL, $\mathrm{P}=0.13$, respectively). The rates of no reflow phenomenon and TIMI thrombus bur- den between the two groups were also similar. Lastly, there was no significant between-group difference in all-cause mortality in the setting of true STEMI excluding OHCA (14\% vs. $13 \%, \mathrm{P}=0.9$ ).

\section{Discussion}

There has been substantial interest in the cardiology community regarding ACS during the COVID-19 pandemic. In both the USA and Spain, centers have reported a decrease in overall cardiac catheterizations and STEMI activations [2, 3]. Multiple reports have also suggested that patients with STEMI are presenting later and experiencing longer DTB times [4]. A recent study out of Italy also reported reductions in cardiac catheterization cases across the spectrum of ACS [5]. Similar to this recently published data, we also observed a significant reduction in STEMI activations during the COVID-19 era. The main driver for this reduction in STEMI activations may be explained by the significant fall in OHCA cases who present to the emergency room. Our local emergency room changed the policy for OHCA during this pandemic, and now requires sustained return of spontaneous circulation of more than $5 \mathrm{~min}$ before the ambulance crew can bring the patient to the hospital. Further studies are necessary to determine whether the reduction in OHCA cases are due to increased out-of-hospital mortality. Furthermore, we found clinically meaningful and consistent trends for reduced catheterization volume in both UA/NSTEMI and true STEMI, though these between-group differences did not reach statistical significance likely due to our modest sample size, which was a limitation to our study. 

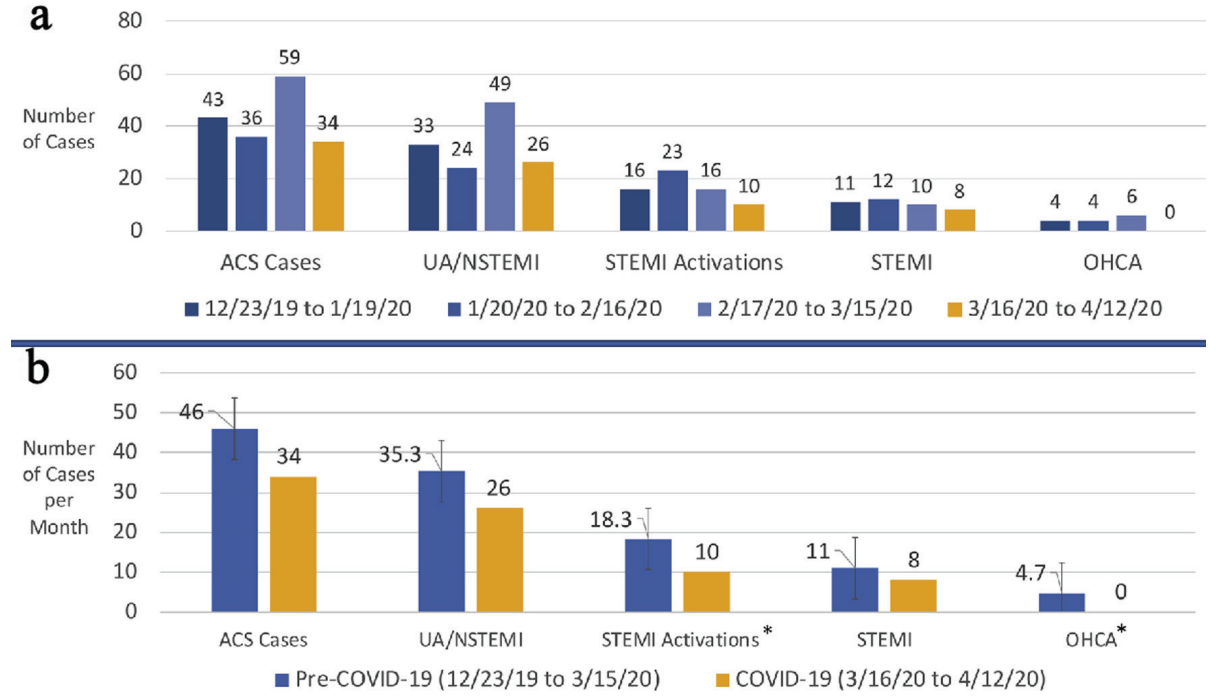

Figure 1. Cardiac catheterization volume in the setting of ACS. (a) The number of cardiac catheterizations for each clinical indication separated by month. (b) The mean number of monthly cardiac catheterizations prior to the COVID-19 era compared with the number of cases during the COVID-19 era. Standard error bars are shown. *Statistically significant difference with a $\mathrm{P}$ value < 0.05. COVID-19: coronavirus disease 2019; ACS: acute coronary syndrome; UA: unstable angina; NSTEMI: non-ST-segment elevation myocardial infarction; STEMI: ST-segment elevation myocardial infarction; OHCA: out-of-hospital cardiac arrest.

While cardiac catheterization volume seems to be reduced during the pandemic, outcomes for these patients presenting with true STEMI are similar to patients presenting with true STEMI prior to the COVID-19 pandemic. Initial markers of cardiac injury for patients with true STEMI in the COVID-19 era were not significantly higher than those with true STEMI before the COVID-19 era, suggesting that patients in our geographic location may not be waiting longer at home before presenting to the hospital for care. Furthermore, the rates of no reflow phenomenon and high thrombus burden, both markers of delayed presentation for STEMI [6], and allcause mortality were not different in our limited number of patients with true STEMI before and during the COVID-19 era. This may be due to the fact that the number of patients infected with COVID-19 in Los Angeles County [7] has not been as high as other regions of the country. Importantly, DTB times did not change despite the addition of extra precautionary measures (e.g., personal protective equipment (PPE)), allaying fears of delayed percutaneous coronary intervention and supporting primary percutaneous coronary intervention as the gold standard even in the COVID-19 era unless PPE is unavailable. Our cardiac catheterization laboratory was quick to implement and streamline a workflow where lab personnel were able to perform cases with appropriate PPE without delay in patient care.

In summary, our data collectively suggest that the initially reported reduction in STEMI activations is also seen across the spectrum of ACS, without a change in outcomes for STEMI cases. Longer-term data coupled with further insights from emergency medical system databases will be needed to further elucidate the mechanisms for the observed changes in cardiac catheterization volume during the COVID-19 era. Larger studies with longer follow-up will also be necessary to determine whether ACS-related outcomes are indirectly altered and worse during the COVID-19 pandemic.

\section{Acknowledgments}

None to declare.

\section{Financial Disclosure}

The authors have no financial disclosure or conflict of interest to disclose.

\section{Conflict of Interest}

The authors report no conflict of interest related to the contents of this manuscript.

\section{Informed Consent}

Given the retrospective nature of the study, informed consent was waived by the UCLA Institutional Review Board.

\section{Author Contributions}

WT designed the study, engaged in data collection, oversaw data collection, reviewed the literature, analyzed and interpreted the data, and drafted the manuscript. RP oversaw data collection, reviewed the literature, contribute to drafts of the manuscript, and provided critical review of the manuscript. 
$\mathrm{RC}$ engaged in data collection, data interpretation, and contributed to drafts of the manuscript. JH engaged in data collection and data interpretation. VF provided critical reviews of the manuscript. OA provided critical review of the manuscript. $\mathrm{RD}$ provided critical review of the manuscript. AR oversaw data collection, reviewed the literature, analyzed and interpreted the data, and provided critical review of the manuscript. MP contributed to the design of the study, analyzed and interpreted data, and provided critical review of the manuscript.

\section{Data Availability}

The authors declare that data supporting the findings of this study are available within the article.

\section{References}

1. National Center for Immunization and Respiratory Diseases (NCIRD) D of VD. Interim guidance for healthcare facilities: preparing for community transmission of COVID-19 in the United States. 2020.

2. Rodriguez-Leor O, Cid-Alvarez B, Ojeda S, MartinMoreiras J, Rumoroso JR, Lopez-Palop R, Serrador A, et al. Impacto de la pandemia de COVID-19 sobre la actividad asistencial en cardiologia intervencionista en Espana. REC Interv Cardiol. 2020;2:82-89.

3. Garcia S, Albaghdadi MS, Meraj PM, Schmidt C, Garberich R, Jaffer FA, Dixon S, et al. Reduction in ST-segment elevation cardiac catheterization laboratory activations in the United States during COVID-19 pandemic. J Am Coll Cardiol. 2020.

4. Tam C-CF, Cheung K-S, Lam S, et al. Impact of coronavirus disease 2019 (COVID-19) outbreak on ST-segment-elevation myocardial infarction care in Hong Kong, China. Circ Cardiovasc Qual Outcomes. 2020;2019(Apr il):2019-2021.

5. De Filippo O, D'Ascenzo F, Angelini F, Bocchino PP, Conrotto F, Saglietto A, Secco GG, et al. Reduced rate of hospital admissions for ACS during COVID-19 outbreak in Northern Italy. N Engl J Med. 2020.

6. Buono A, Gori T. No-reflow phenomenon in acute myocardial infarction: Relieve pressure from the procedure and focus attention to the patient. Int J Cardiol Heart Vasc. 2019;24:100417.

7. COVID-19 surveillance dashboard [Internet]. Los Angeles County Department of Public Health. 2020. Available from: http://dashboard.publichealth.lacounty.gov/ covid19_surveillance_dashboard/. 